\title{
Context-Aware IPTV
}

\author{
Songbo Song ${ }^{1}$, Hassnaa Moustafa ${ }^{1}$, and Hossam Afifi $^{2}$ \\ ${ }^{1}$ France Telecom R\&D (Orange Labs), Issy les Moulineaux, France \\ \{songbo.song, hassnaa.moustafa\} dorange-ftgroup.com \\ ${ }^{2}$ Telecom \& Management South Paris (Institute Telecom), Evry, France \\ \{hossam.afifi\}@int-edu.eu
}

\begin{abstract}
The advances in IPTV technology enable a new user-centric and interactive TV model, in which context-awareness concept is promising in making users' interaction with the TV dynamic and transparent. In this paper we present several existing contributions that employ context-awareness concept to allow interactive services. Some of these contributions directly focus on TV, while others are proposed for specific NGN services. We analyze these contributions based upon some requirements that we define for enabling advanced IPTV services personalization.
\end{abstract}

Keywords: NGN, IPTV, Personalized Services, User-Centric IPTV.

\section{Introduction}

The new TV model will allow users not only to access new services and functionalities from their providers, but also to become active parts in the content creation and distribution process. With the consolidation of services, such as network Time Shifting (nTS) and network Personal Video Recorder (nPVR), users are allowed to record their own content and could also make them available to other users. The Next Generation Network (NGN) approach in coupling IPTV with IP Multimedia System (IMS) allows for services' convergence through using the IMS common architecture in providing a platform for interactive TV services. Although the rapid advancement in interactive TV technology (including IPTV and NGN technologies), IPTV service personalization is still in its infancy (for instance, content recommendation is not totally user-centric, choosing the desired channel in a manual mean could disturb the viewer). Context-awareness paradigm is promising in simplifying the viewer's life, through allowing content adaptation according to the user's needs and to the surrounding environment. We notice an increasing research trend for context-awareness IPTV systems, where promising applications and services' enhancements are expected. In this paper we focus on context-aware TV and NGN services.

The remainder of this paper is organized as follows. Section 2 defines a number of requirements that should be satisfied for TV services personalization. Section 3 presents the related contributions on context-aware interactive TV and context-aware NGN services based on IMS, analyzing them with respect to those defined requirements. We end the paper in Section 4 through giving a conclusion for the existing contributions, highlighting our future research work. 


\section{Requirements for TV Services Personalization}

In this section, we define a number of requirements for analyzing the existing contributions on context-aware TV and NGN services. These requirements help in evaluating the suitability and adaptability of each contribution to the interactive TV environment.

Context information domain (types): The architecture requirements for IPTV include four domains: consumers, network providers, service providers, and content providers. Based on these domains, we define our context information domains for TV environment as: user context, device context, network context, and service context. The user context and device context belong to the "consumer domain"; the network context belongs to the "network domain"; the service context presents the information from the "service and content domain".

Context information extension: Context information types and treatment methods can be designed either for general services or for special type of services. However, the extension/adaptation of the context information types and treatment methods to TV service is an important aspect which determines the possibility to integrate contextawareness into the NGN TV architecture.

Distributed versus centralized system: A distributed context-aware system is constituted by several entities that have the capacity to acquire, process, and store the context information. These entities can cooperate with each other to provide the context-aware services. In distributed systems, it is difficult to have a global view of the context information and the calculation capacity is limited to the distributed terminals capacities. A centralized context-aware system treats and stores the collected context information in a centralized server; however, the frequent communication between the devices and the server needs adequate network resources.

Support for mobility: Mobility is an important feature that should be supported in modern TV systems to allow the user to enjoy his personal TV content any time and any where. This requires context-aware TV systems to integrate appropriate mechanisms for continuous devices discovery and continuous location discovery for the user, in order to support the seamless TV service transfer and continuity.

Support for nomadism: With the new model of TV systems, users should be able to enjoy their personal TV content from anywhere. So, we define a new scope for nomadism to allow users to access their personal TV content and to pay through their own bill while passing by other's networks. To support such nomadic services, context-aware TV systems, beside acquiring the user identification should also be capable of acquiring other context information 'location, which connectivity, ...'.

Support for privacy protection: The privacy protection is the capability of a user or group of users to secure their personal information. Since any context-aware system is concerned to a great extent with personal information (user context), the privacy protection is necessary. Privacy protection in TV environment is mainly related to the following points: controlling the personal information sharing; not publishing the content information that the user is accessing (e.g., mastering the content sharing); user context storage and manipulation among authorized entities. 
Performance and feasibility of implementation: Some context-aware functions may be not easy to implement due to the need of lot of processing power, calculation and storage capacity. Terminals capacity should then be considered in context-aware TV systems. Furthermore, the used protocols and the underlying architectures for contextaware TV systems should be compatible with the existing standards (especially those related to NGN).

Support for personalization: TV service personalization allows the content to meet the expectation/preference of the user. This refers to two points: identification of the user and finding out what content the user prefers. Furthermore, the support of mobility, nomadism and privacy protection present important requirements for achieving the provision of content in a personalized and user-centric manner.

Co-existence of multiple actors: Since modern TV services (especially IPTV) include multiple actors such as network operators, service providers, and content providers, the co-existence of these different actors should be assured by any context-aware TV system. Although IMS can provide some functional support to context-aware service, the dependence on IMS architecture has some implementation restrictions, especially when we look for compatibility among the various actors. Furthermore, the managing and sharing of context information among the various actors is not an easy task.

\section{Contributions on Context-Aware Based Interactive Services}

In this section, we present and discuss some existing contributions on Interactive TV (ITV), which employ context-awareness in order to enhance the user interaction with the TV environment. Other contributions are related to context-aware based NGN IMS services, which are not explicitly related to TV services, but we also discuss these contributions showing their suitability in TV environments based on our defined requirements.

A context-aware based advertisements (ads) insertion system is proposed in [1]. This work is based on the aggregation of past sequence of individual contexts (i.e. past viewing) and the association of the current user context to those past contexts in order to determine the most appropriate advertisement. The used context includes user's location and activity, user/device/content/event identity, and time and is organized as Current context (CCo) and Analyzed/Aggregated Context (ACo). The ACo is the analyzed and aggregated past context sequences presenting the past history of the CCo. This proposed ITV system is mainly targeting home networks and is realized through four entities: User Identification (UId), Context Derivation (CoD), Bulk Ad Retrieval (BAR) and Context-aware Ad Selection and Insertion (CaASI). This work presents a centralized system since the context information treatment is realized on the Set-Top-Box (STB). However the processing capacity of the STB limits its performance. No context discovery and service transfer mechanism are provided in this work, thus leading to a limited mobility support. Although a Channel Surfing Analysis algorithm is proposed to identify the users watching the TV, it is not very accurate since it is based on some hypothesis and also fails to detect visitors which restrict the nomadism. This work does not consider the privacy protection. The system in this work is in the application level and not dependent on the specific network architecture, so it can be implemented on any NGN platform. 
An ITV system is proposed in [2] through applying context-awareness, considering the context information of users, terminals, network and content. Software Agents are implemented on top of physical devices for context acquisition, treatment and storage, composing a distributed ITV system. The agents' capacity in discovering other agents allows the devices to discover each other and the 'active network' used in this work which can decide the best way to route the TV content according to the context (traffic condition, user's location, etc.). However, the communication capacity between agents (for example the communication scope) is limited which impacts the mobility support. The lack of an identification mechanism and the distributed storage of context information stand as obstacles for nomadism. Furthermore, we notice that this work, does not apply any context inference mechanism or dynamic user experience analysis, so the service personalization is limited. Since this work is not dependent on a specific under-lying architecture, it can support co-existence of different actors.

In [3], a client/server based context-aware framework is proposed to enhance the TV services through including TV Set automatic control and personalized content recommendation. This framework is composed of two parts: Service Agent Managers (SAM) and the CAMUS Server. The SAM contains Service Agents (SA) and each SA detects the context information from the dedicated device (sensor or equipment) and forwards it to CAMUS server. The latter treats the context information through context manager which is responsible of the context representation, inference and storage as well as discovering the ongoing application through the service request. Then tasks are generated through a task manager included in CAMUS according to the stored rules, context information and ongoing applications. The SA then receives task commands from CAMUS and controls the device according to the commands. The context information mentioned in this work concerns the user, the devices and the content, and can be extended by adding new SAs. The centralized storage of context information and RFID based identification make the nomadism possible, but limited in the server implementation scope. Since this work does not consider the network context and does not allow the service transfer (no session control functionalities exist), the mobility support is limited. And since this work is not limited to a specific underlying architecture, it could allow for the co-existence of multiple actors; however the privacy protection is not considered.

In [4], the authors integrate the presence service into the IMS based emergency service aiming to provide context-awareness. The context information mentioned in this work is user's physiological information (as blood pressure) and environment information (as sound level) and is acquired by Wireless Sensor Networks (WSNs). It is then relayed to sensor gateways acting as interworking units between the WSN and the IMS core network. The gateway is considered as the presence external agent (PEA, an agent used to publish presence information in presence service), where it collects the information, processes it (e.g. through aggregation and filtering), and then forwards it to the presence server that plays the role of the Context Information Base (CIB) responsible for context information storage, management and dissemination. The two entities E(Emergency)-CSCF and location retrieval function (LRF) which are used to establish the emergency session in traditional emergency services are merged into the CIB. The Context retrieval function (CRF) at the service provider side retrieves different context information about the user. This presence service based 
system is centralized, where the presence agent allows the extension of context information, however no context information inference mechanism is provided which limits the service personalization. This work could benefit from the privacy protection in the classical presence service to assure users' privacy. The user's identification and authentication follow the IMS approach, attaching the user identification to that of devices which restricts the nomadism. Although the WSNs can discover devices, the lack of service's transfer among different devices limits the mobility support.

In [5], the IMS Push to Talk over Cellular (PoC) service is enhanced by adopting context-awareness through employing the presence service to master the storage, management and distribution of the context information. Compared to traditional PoC architecture, this proposed architecture adds a context-aware collector in the environment to collect the context information, and adds the presence service user agent in the PoC terminals to forward the context information to the presence server. Furthermore in the Group List Management Server (GLMS), besides the contact list of a certain user, stores the context-aware rules. Through the rules and the context information in the presence server the PoC server can infer which user will be added in the communication session. Although the context information considered in this work is limited to the user context, it can be extended through implementing the contextaware collector in different environments. The presence user agent located in each device helps the system to discover the devices, while the context collector can continuously acquire the user's location. The PoC server can also handle the session transmission allowing the system to support mobility. We notice that the user's identification follows, attaching the user identification to that of devices, which limits the nomadism. The privacy protection can be carried out through presence service.

In [6], a general context-aware service enabler based on the core IMS architecture is proposed. This contribution targets general IMS services and does not specify the context information domains. The enabler continuously communicates with the context sources which are located in the environment to acquire the context information and stores it in the Context Database. When the application server receives a request from the user, it firstly contacts the Context Query Engine which provides an abstract interface to the context-aware service enabler for applications to extract the desired contexts, where a Query Generator function creates the query according to the service description and forwards it to the Reasoning Engine through a query request. The Reasoning Engine includes a Reasoner function used to infer high-level context information from low level ones based on the rules stored in the Rule Manager and a Query Execution function treating the query sent by the Query Engine based on the context information in the context database and the reasoning results. At last, according to the query response, the Service instructor generates the service instruction used to configure the services. This centralized system treats the context information on a server which has enough capacity to provide adequate high-level user context information. Although no user's identification mechanism is provided, the context-aware enabler can identify the user based on the user context information, so the nomadism can be supported. Since the context sources can discover the devices and the session control is provided by the IMS, this work can support mobility. But this work does not mention the privacy protection. In order to support interactive TV service, the service functions (like content recommendation function) should be added. 


\section{Summary and Conclusion}

In this paper, we focus on context-aware TV system (with a special interest on IPTV). Through our study and analysis to the different related contributions discussed in this paper, we notice that no existing contribution could satisfy service personalization in a complete and adequate manner. For ITV, most of the existing works treat the TV services personalization through only content recommendation and targeted advertisement insertion. However, the provision of content in a personalized and usercentric manner taking into consideration mobility and nomadic situations is not yet resolved. From the context-aware based IMS architecture, we can discover that the context-aware concept enhances the personalized information treatment in IMS. However, how to use the acquired context information to realize the personalized IMS based IPTV services is still a challenge. Furthermore, the privacy protection is another issue that is not resolved in the existing contributions, where user-context information should not be a way that threatens the user privacy.

Our next step is to benefit from this study and to integrate a context-awareness system into an IPTV/IMS platform that we have. Our future context-aware IPTV system will satisfy the different requirements that we defined in order to enhance the IPTV services and fulfill a number of use-cases that are promising for the network operator in opening new business opportunities.

\section{References}

1. Thawani, A., Gopalan, S., Sridhar, V.: Context Aware Personalized Ad Insertion in an Interactive TV Environment. In: 4th Workshop on Personalization in Future TV (2004)

2. Santos, J.B.D., Goularte, R., Faria, G.B., Moreira, E.D.S.: Modeling of user interaction in context-aware interactive television application on distributed environments. In: 1st Workshop on Personalization in Future TV, Sonthofen (2001)

3. Moon, A., Kim, H., Lee, K., Kim, H.: Designing CAMUS based Context-Awareness for Pervasive Home Environments. In: International Conference on Hybrid Information Technology, Cheju Island, pp. 666-672 (2006)

4. El Barachi, M., Kadiwal, A., Glitho, R., Khendek, F., Dssouli, R.: An Architecture for the Provision of Context-Aware Emergency Services in the IP Multimedia Subsystem. In: Vehicular Technology Conference, Singapore, pp. 2784-2788 (2008)

5. Hsu, J.M., Lain, W.B., Chan, C.C., Huang, Y.M.: Implementation of IMS-based PoC Service with Context-Aware Interaction. International Journal of Multimedia and Ubiquitous Engineering 3, 25-43 (2008)

6. Kim, J., Jeong, J., Nam, S.M., Song, O.: Intelligent Service Enabler based on ContextAware in Next Generation Networks. In: International Symposium on Parallel and Distributed Processing with Applications, pp. 802-906 (2008) 